\title{
A Conceptual and Operational Review of the Negative Financial Health Terminology and Constructs
}

\author{
Amanda Wuth ${ }^{1} \&$ Magdalena Cismaru ${ }^{2}$ \\ ${ }^{1}$ Gordon S. Lang School of Business and Economics, University of Guelph, Guelph, Canada \\ ${ }^{2}$ Faculty of Business Administration, University of Regina, Regina, Canada \\ Correspondence: Magdalena Cismaru, Faculty of Business Administration, University of Regina, Regina, \\ Saskatchewan, S4S 0A2, Canada.
}

Received: February 22, 2021

Accepted: March 12, 2021

Online Published: March 16, 2021

doi:10.5539/ibr.v14n4p1

URL: https://doi.org/10.5539/ibr.v14n4p1

\begin{abstract}
Financial (di)stress is widespread and an important topic for research by a variety of organizations and disciplines. However, different terms are being used in different disciplines in academia, by organizations, and by consumers. This paper illustrates various terms used to describe negative financial health, provides their incidence in several academic databases and Google searches, provides definitions used in studies, identifies scales of measurement, assesses if new scales are being developed and if they have validity, and identifies if measures of negative financial health constructs include objective, subjective, or both measures. The study ends with specific recommendations for researchers from academia and practitioners worldwide. This article reviews financial negative health terminology and constructs, and attempts to shed light on similarities and differences among the terms, to allow for better knowledge translation and integration.
\end{abstract}

Keywords: economic hardship, financial distress, economic deprivation, financial strain, financial stress

\section{Introduction}

More than one-third of adults in the USA, Canada, Australia and New Zealand are struggling financially and experience heightened levels of material hardship (Muir et al., 2016; Seymour Management Consulting Inc., 2017). Increasingly, more people are expected to experience financial stress in the future (American Psychological Association [APA], 2017). In addition, measures taken in countries to slow the spread of COVID-19 have also exacerbated individual negative financial health and its consequences (e.g., Mann, Krueger, \& Vohs, 2020; Turcotte \& Hango, 2020; Witteveen, 2020). Financial (di)stress has damaging consequences for individuals and their families ranging from declining health to divorce and unsecured debt (Cismaru \& Wuth, 2019) prompting academics and organizations to study and try to eliminate this problem.

Due to the impact financial (di)stress has on individuals and society, personal negative financial health is studied across disciplines (e.g., finance, psychology, economics, sociology, marketing) (APA, 2017; Athreya, Mustre-del-Río, \& Sánchez, 2017; Australian Psychological Society [APS], 2015; Park, Heo, Ruiz-Menjivar, \& Grable, 2017) and by a mixture of organizations (universities, governments and financial institutions) (i.e., Consumer Financial Protection Bureau [CFPB], 2015; Gallup Inc., 2018a; Muir et al., 2016; O’Neill, Prawitz, Sorhaindo, Kim, \& Garman, 2006; Seymour Management Consulting Inc., 2017). Various government organizations such as the National Australia Bank have taken interest in their citizens' financial health, examining the prevalence of financial stressors and financial resilience, and are working toward advancing financial resiliency in their clients (e.g., CFPB, 2015; Muir et al., 2016). Similarly, findings from the Seymour Management Consulting Inc. (2017) report suggest Canadians want their financial institutions to provide more financial resources and play a larger role in helping them with their financial health, hence more banks in Canada taking an active role toward promoting financial well-being. This high level of involvement by numerous institutions and organizations is reflected also in the numerous social marketing initiatives run by both government and financial institutions with the declared aimed of promoting financial well-being in their citizens or clients (see Cismaru \& Wuth, 2019 for a review).

With several disciplines and organizations working on tackling financial stress, a variety of terms have been used, i.e., financial stress, financial distress, financial strain, financial pressure, economic hardship, economic 
deprivation, income inadequacy. Some of these terms mean exactly the same thing, some terms are similar but not identical, and some terms overlap in some respects and are different in other respects, creating confusion and making knowledge translation difficult. Indeed, different terminology is used across disciplines as well as by researchers and industrial practitioners. Financial terminology has been redefined and new scales have been constructed, e.g., financial well-being (CFPB, 2015), financial control (Gallup Inc. 2018a), financial wellness and financial resilience (Seymour Management Consulting Inc., 2017). However, authors from one discipline might not consult research specific to another discipline, or even look at reports from industry, which themselves may not build on studies from the academic field. Industrial reports may not be available through academic databases, may not be peer-reviewed, and may not refer to scientific terms and/or specify whether a new developed scale was validated (e.g., Gallup Inc., 2018b). These facts are problematic, as a variety of terms and measures are used or introduced, creating confusion for someone attempting to integrate knowledge.

Therefore, this paper fills the need for a review of negative financial health terminology and constructs to shed light on similarities and differences among the terms and measurements, and allow for better knowledge translation and integration across academic disciplines, and between academia and government and industry. Recent articles indicate a need for a more dynamic knowledge translation, especially between academics and practitioners (see Levit \& Cismaru, 2020).

\section{Literature Review}

Conceptual definitions provide the basis for operational definitions and determination of how to empirically assess constructs (Bernard, 2012). While research studies diligently report what questionnaires were used (operational definition), many studies fail to concurrently provide an understanding of what is being assessed (conceptual definition). Conceptual terms are used to clarify specific, scientific terms, and ensure readers understand the term in the same way that the authors did (Dumont \& Wilson, 1967; Lester \& Lester Jr., 2015; Newman, Ridenour, Weis, \& McNeil, 1997). However, understanding of definitions is further confounded by the inconsistent use of specialized terminology, proxy terms, and unspecified synonyms (Bernard, 2012). Using unspecified synonyms is problematic when there is no scientific thesaurus readily available or the author understands a specific construct differently from their audience. In order to develop a single conceptual framework, that bridges disciplinary boundaries, it is vital that the same terms are used to discuss concepts (Simon, 2014). It is therefore necessary to further clarify terminology associated with individual experiences of negative financial health.

Indeed, analogous relationships exist between financial stress, financial strain, financial hardship, economic deprivation, economic pressure, and perceived income inadequacy (Kim \& Garman, 2003; Sinclair \& Cheung, 2016). Financial distress, economic stress, and economic strain have also been defined similarly within the literature (e.g., Mills, Grasmick, Morgan, \& Wenk, 1992; Voydanoff, 1990). However, scientific and non-scientific terms are vastly different.

Non-scientific terms are widely available in dictionaries and thesauruses. The definitions and synonyms provided by one publisher are similar to those provided by another publisher. In contrast, scientific terms are jargon, and their definitions are spread across various locations. They are often created by researchers to explain latent constructs, and the term being used to describe a concept may differ from the concept that a tool was designed to assess. This poses a problem for researchers: how do you effectively communicate your findings in a manner that anyone from any background can understand the results? How do you bridge the systematic barrier of construct proliferation?

This paper will identify negative financial health terminology, present its use in various databases and on Google by both academics and general public, look for definitions of these terms in academic studies, identify scales used in studies and if new developed scales had validity, and also, if the measures include both subjective and objective components. Ultimately this study could provide an initial lexicon for understanding the breadth of terminology involved in studying negative financial health.

Financial health can be broadly described through positive terms, i.e., financial well-being, and negative terms, i.e., financial stress, financial di(stress), financial strain, financial hardship, etc. Recent definitions of financial well-being include a current and a future component but also refer to individuals considering their objective financial situation as being adequate for their needs and wants and their perception of being able to maintain their current financial situations and actualize desired financial states (subjective financial situation) (Brüggen, Hogreve, Holmlund, Kabadayi, \& Löfgren, 2017; CFPB, 2015; Kempson, Finney, and Poppe 2017; Netemeyer, Warmath, Fernandes, \& Lynch Jr., 2017). Hence an objective and a subjective component (measures) focus on the positive terms has emerged in the financial health literature. 
In contrast, the negative terminology literature is not so advanced. For example, one of the newest definitions of financial distress refers to it as an accumulation of financial stressors that limits one's objective situation and ability to maintain current and future financial needs and wants (Brüggen et al., 2017; Lajuni, Bujang, Karia, \& Yacob, 2017). This definition does not appear to include a subjective component. This could create a discrepancy between positive and negative financial terminology which can be problematic as the influx of new terms, definitions, and measures of the positive financial terms may obscure overall understanding of the negative one. As more articles expand negative financial terminology, given the importance of increasing financial resiliency through the transdisciplinary objective of understanding negative financial health constructs, construct proliferation is a wide-spread problem and a clarification of the terminology associated with negative financial health is needed. Hence the focus of this paper on clarifying negative terms such as financial (di)stress.

However, despite differences in the conceptualization of personal negative financial health terms, we seek to identify similarities and trends in how these terms are operationalized, that parallel the operationalization of financial well-being terms (e.g., Kempson et al., 2017). For example, like financial well-being, financial distress may be operationalized through objective assessments, subjective assessments, or both. Objective assessments refer to items that are verifiable and based on fact such as amount of monthly savings or total savings, and amount of debt (Muckler \& Seven, 1992). Objective assessments are less susceptible to differences in perception, cognition, and experience because they are values individuals should be able to calculate. Alternatively, subjective assessments are distorted by perceptions, cognitions, and experiences unique to the individual (Muckler \& Seven, 1992). In the context of financial distress, subjective assessments may acknowledge that two individuals with the same debts and credits can report different levels of financial distress because of their perception of the financial resources they want and those which they currently have (e.g., O'Neill et al., 2006). Subjective assessment recognizes personal insight and the need to accommodate for individual differences since perceived financial distress may differ from an objective assessment of financial distress (Muckler \& Seven, 1992). Objective and subjective measures consequently assess different aspects of one's financial situation, and provide different sources of information.

Previous work in this area includes a conceptual review of negative financial health terms conducted by Sinclair and Cheung (2016) and a conceptual review of financial stress conducted by Davis and Mantler (2004). Sinclair and Cheung focus on providing measurement recommendations and highlighting the need for more research within the field of occupational health psychology. Davis and Mantler (2004) also include a conceptual review of financial stress, differentiate between financial stress and economic hardship, and discuss the effects of financial stress on people, families, and society. They also provide an excellent starting point for understanding financial stress and similar concepts; however, many relevant constructs were not discussed in their paper and the field has expended substantially since the early 2000 s.

Although many negative financial health constructs exist, throughout this paper we will generally refer to this collection of constructs as personal or individual negative financial health. Individual negative financial health is broadly defined as any state wherein individuals perceive themselves as experiencing one or several negative financial events, financial issues, or financial stressors, which makes them unable to maintain their current or achieve a desired financial state (e.g., Joo, 1998; Kim \& Garman 2003; Lim, Heckman, Montalto, \& Letkiewicz,. 2014; O'Neill et al., 2005; O'Neill et al., 2006). This broad label subsumes economic-, financial-, and income-based terms, such as economic hardship, financial distress, and economic deprivation.

\section{Method}

\subsection{Terminology Identification and Selection}

Keyword literature searches for articles with negative health terminology were inspired by Sinclair and Cheung (2016) and were conducted in the following databases: Google Scholar, JStor, ProQuest, PsycInfo, PubMed, Sage, Science Direct and Wiley Online Library. Keywords used include economic hardship, financial distress, economic deprivation, financial strain, financial stress, financial issues, financial hardship, economic pressure, economic stress, financial pressure, and financial strain. New financial terms were added throughout the literature review process. Counts from 27,642 articles were conducted to determine the prevalence of each negative financial term, by database (see Table 1).

In order to gain greater understanding of how the general public engages with negative financial health terms, keywords identified from the literature search were also analysed with Google Trends (Google, 2021). Google Trends allows researchers to compare the relative interest in different search terms across time and geographic region. The relative popularity of a single search term is calculated by dividing a geographic total by the total searches conducted in that geographic area within a given timeframe. The relative popularity score of each term 
is then scaled from 0 to 100 to indicate interest in the search term over time (Google, 2021). Google Trends have been widely utilized in academic research to forecast behavior and provide unique insights into social phenomenon (reviewed in Jun, Yoo, \& Choi 2018). In this paper, global non-real time Google search data was reviewed for a five-year time period (January 2016 to January 2021). The keywords identified from the literature searches were compared from the most to least popular academic terms. Since there is a five-term limit for comparisons, the top term from each search (i.e., financial hardship, financial stress, and financial distress) was included in a subsequent comparison to determine which term was most popular in English speaking countries.

Table 1. Key term search of financial distress and related concepts, citations through October 2018

\begin{tabular}{|c|c|c|c|c|c|c|c|c|c|}
\hline Database & JStor & $\begin{array}{c}\text { ProQue } \\
\text { st }\end{array}$ & $\begin{array}{l}\text { PsycInfo/Ov } \\
\text { id }\end{array}$ & $\begin{array}{c}\text { PubMe } \\
\text { d }\end{array}$ & $\begin{array}{c}\text { Sage } \\
*\end{array}$ & $\begin{array}{l}\text { Science } \\
\text { Direct*** }^{*}\end{array}$ & $\begin{array}{c}\text { Wiley Online } \\
\text { Library* }\end{array}$ & Sum & $\begin{array}{c}\text { Percentag } \\
\text { es }\end{array}$ \\
\hline Economic Hardship & 240 & 1753 & 764 & 676 & 140 & 231 & 206 & 4010 & $14.54 \%$ \\
\hline $\begin{array}{r}\text { Financial Distress } \\
\text { Economic }\end{array}$ & 310 & 1777 & 215 & 222 & 64 & 634 & 391 & 3613 & $\begin{array}{l}13.10 \% \\
10.38 \%\end{array}$ \\
\hline Deprivation & 147 & 1111 & 483 & 654 & 103 & 184 & 182 & 2864 & \\
\hline Financial Strain & 68 & 786 & 735 & 750 & 92 & 160 & 143 & 2734 & $9.91 \%$ \\
\hline Financial Stress & 90 & 1033 & 546 & 516 & 86 & 269 & 185 & 2725 & $9.88 \%$ \\
\hline Financial Issues & 61 & 1013 & 423 & 606 & 80 & 215 & 147 & 2545 & $9.23 \%$ \\
\hline Financial Hardship & 59 & 718 & 414 & 612 & 81 & 131 & 122 & 2137 & $7.75 \%$ \\
\hline Economic Pressure & 111 & 738 & 201 & 306 & 47 & 370 & 89 & 1862 & $6.75 \%$ \\
\hline Economic Stress & 109 & 727 & 382 & 285 & 68 & 138 & 125 & 1834 & $6.65 \%$ \\
\hline Financial Pressure & 46 & 379 & 90 & 235 & 41 & 208 & 52 & 1051 & $3.81 \%$ \\
\hline Economic Distress & 85 & 444 & 128 & 83 & 47 & 55 & 52 & 894 & $3.24 \%$ \\
\hline $\begin{array}{r}\text { Economic Strain } \\
\text { Economic }\end{array}$ & 30 & 315 & 187 & 125 & 33 & 43 & 48 & 781 & $\begin{array}{l}2.83 \% \\
1.72 \%\end{array}$ \\
\hline Adversity & 25 & 184 & 99 & 90 & 19 & 32 & 26 & 475 & \\
\hline Income Inadequacy & 2 & 18 & 18 & 15 & 1 & 2 & 5 & $\begin{array}{r}61 \\
2758\end{array}$ & $0.22 \%$ \\
\hline Total & $\begin{array}{c}1383 \\
5.01\end{array}$ & 10996 & 4685 & $\begin{array}{l}5175 \\
18.76\end{array}$ & $\begin{array}{l}902 \\
3.27\end{array}$ & 2672 & 1773 & 6 & \\
\hline Percentages & $\%$ & $39.86 \%$ & $16.98 \%$ & $\%$ & $\%$ & $9.69 \%$ & $6.43 \%$ & & \\
\hline
\end{tabular}

Notes: $*=$ Key terms only in abstract. $* *=$ Key terms in title, abstract, or keywords.

\subsection{Article Identification and Selection}

For the analysis of conceptual and operations definitions, following the methodology of Cismaru and Wuth (2019) and Sinclair and Cheung (2016), the top 50-100 results generated by each database and search engine were considered for inclusion. We then established several inclusion and exclusion criteria. Articles were included if they were peer-reviewed research or review papers published in academic journals. Published working papers were also considered for inclusion. Articles were excluded if a key financial term was not present in the abstract, title or keywords; they were not peer-reviewed; they were not published in a journal; or published in English. A final sample of 37 articles were included for analysis of conceptual and operational definitions.

\section{Results}

\subsection{Negative Financial Health Terminology}

\subsubsection{Incidence}

Within academic spheres, as shown in Table 1, 27586 incidences of negative financial terms were identified. In terms of the presence of negative financial health terms in the specific databases, almost $40 \%$ were found in ProQuest, followed by $19 \%$ in PubMed, $17 \%$ in PsychInfo/Ovid, approximately $10 \%$ in Science Direct, and in Wiley Online library, JStor and Sage with about $6.5 \%, 5 \%$ and $3 \%$ respectively.

A total of 14 negative financial health terms were identified and counted: economic hardship (4010 times), financial distress (3613 times), economic deprivation (2864 times), financial strain (2734 times), financial stress (2725 times), financial issues (2545 times), financial hardship (2137 times), economic pressure (1862 times), economic stress (1834 times), economic distress (894 times), economic strain (781 times), economic adversity (475 times) and income inadequacy (61 times). Three terms, economic hardship, financial distress and economic deprivation appeared more frequently than other negative financial health constructs, reaching $14.54 \%, 13.10 \%$ and $10.38 \%$ respectively of the total number of times all terms were used. All three terms were favored across 
multiple databases.

Economic hardship was the most commonly used term across PsycInfo/Ovid and Sage databases, while financial distress was the most commonly used term within JStor, ProQuest, Science Direct, and Wiley Online Publishing. Economic hardship was the second most commonly used term across four other databases, while financial distress was either most commonly used or moderately used (i.e., ranked eighth or tenth). Two other terms, financial strain and financial stress, were used in almost $10 \%$ of cases, whereas financial issues and financial hardship were used in about $9 \%$ of cases, respectively $8 \%$ of cases. Five terms, financial pressure, economic distress, economic strain, economic adversity, and income adequacy, reached less than $5 \%$ each out of the total number of uses among the 14 identified terms. Income inadequacy was the least commonly used term across all databases with only $0.22 \%$.

Outside of academia, worldwide, general members of the public are more likely to use financial hardship, financial stress, or financial distress when conducting Google searches for information on negative financial health (Google 2021; see Figure 1). When compared with financial stress and distress, financial hardship was the preferred search term used by people in all the English speaking countries as follows: New Zealand (100\%), Australia (92\%), Canada (64\%), the United States (63\%), and the United Kingdom (52\%; see Table 2).

Table 2. Relative proportion of preferred negative financial health Google search term by region (January $2016-$ January 2021)

\begin{tabular}{cccc}
\hline Country & Financial Hardship & Financial Stress & Financial Distress \\
\hline Australia & .92 & .06 & .02 \\
Canada & .64 & .25 & .11 \\
New Zealand & 1.00 & .00 & .00 \\
United Kingdom & .54 & .29 & .17 \\
United States & .63 & .28 & .09 \\
\hline
\end{tabular}

Notes: $1.00=100 \%$ of searches.

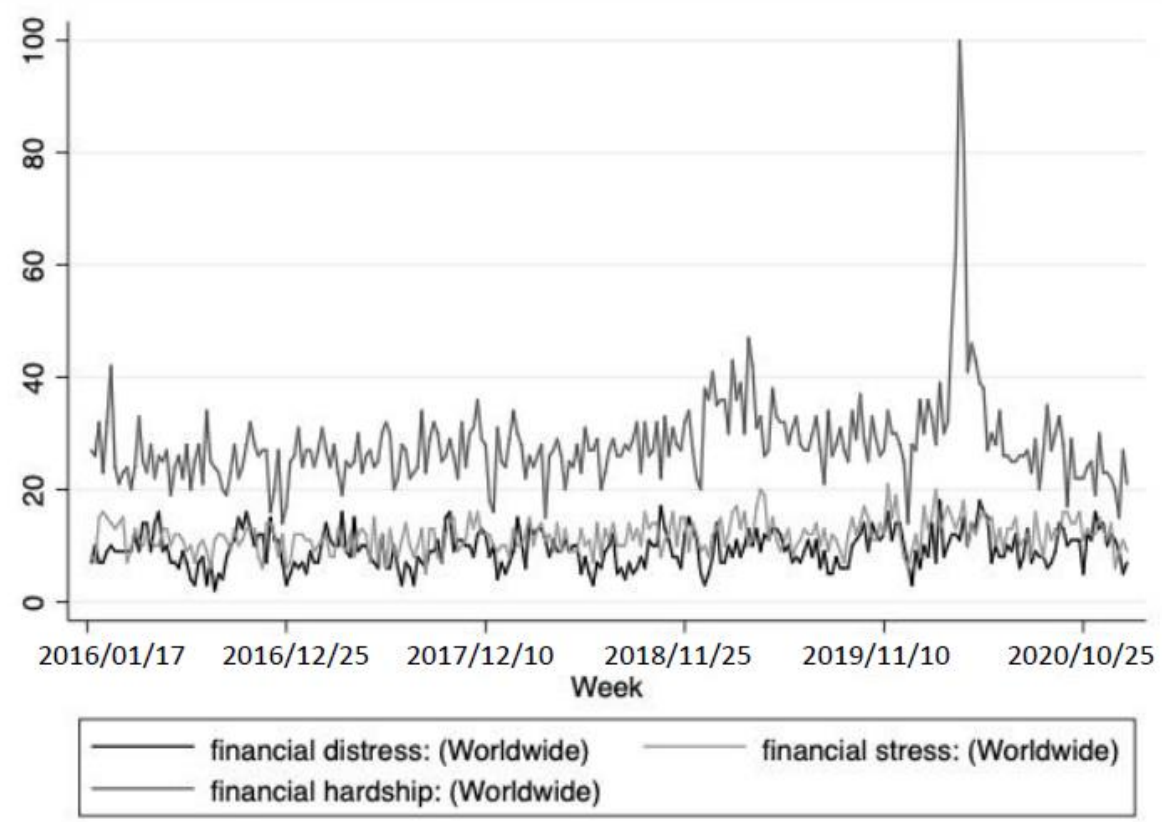

Notes: The maximum interest in the search term financial hardship coincides with the initial implementation of many COVID-19 state of emergency and lock-down protocols.

Figure 1. Relative interest in global negative financial health Google search term (January 2016 - January 2021) 


\subsubsection{Definitions}

As shown in Table 3, in 15 out of 37 studies, no definition of the negative financial health terms is provided by the authors.

\begin{tabular}{|c|c|c|c|c|c|c|c|}
\hline \multirow[t]{2}{*}{ Reference } & \multirow[t]{2}{*}{ Term } & \multicolumn{2}{|l|}{ Conceptual Definition } & \multirow[b]{2}{*}{$\begin{array}{l}\text { New Scale } \\
\text { Developed }\end{array}$} & \multicolumn{3}{|c|}{ Operational Definition } \\
\hline & & Examples & Scale & & Scale Validity & Objective & Subjective \\
\hline $\begin{array}{l}\text { Aldana and } \\
\text { Liljenquist, } 1998\end{array}$ & financial strain & $\begin{array}{l}\text { "Composed of the cognitive, emotional, } \\
\text { and behavioral response to the experience } \\
\text { of financial hardship" (p. 11) }\end{array}$ & $\begin{array}{l}\text { Financial Strain } \\
\text { Survey }\end{array}$ & Yes & $\begin{array}{l}\text { Convergent } \\
\text { validity } \\
\text { measured with } \\
\text { principle } \\
\text { component } \\
\text { analysis } \\
\text { (PCA) } \\
\end{array}$ & $\begin{array}{l}\text { roughly } 20 \% \\
\text { of items }\end{array}$ & $\begin{array}{l}\text { roughly } 80 \% \text { of } \\
\text { items }\end{array}$ \\
\hline $\begin{array}{l}\text { Áslund, Larm, Starrin } \\
\text { and Nilsson, } 2014\end{array}$ & financial stress & $\begin{array}{l}\text { "The persistent inability to afford the basic } \\
\text { necessities of life" (p. 1) }\end{array}$ & $\begin{array}{l}\text { Adapted Finances- } \\
\text { Shame Model (from } \\
\text { Starrin, Aslund and } \\
\text { Nilsson, 2009) }\end{array}$ & $\begin{array}{l}\text { Adapted } \\
\text { from } \\
\text { previous } \\
\text { scale } \\
\end{array}$ & Face validity & $\begin{array}{l}100 \% \text { of } \\
\text { items }\end{array}$ & $\mathrm{X}$ \\
\hline $\begin{array}{l}\text { Athreya, Mustre-del- } \\
\text { Rio and Sanchez, } \\
2017\end{array}$ & $\begin{array}{l}\text { financial } \\
\text { distress }\end{array}$ & $\begin{array}{l}\text { "Situations in which the household } \\
\text { remains susceptible to any deviation of } \\
\text { income from its ex-ante expectation" (p. 3) }\end{array}$ & $\begin{array}{l}\text { Equifax data (no } \\
\text { scale) }\end{array}$ & $\begin{array}{l}\text { Not } \\
\text { applicable }\end{array}$ & Not applicable & $\begin{array}{l}100 \% \text { of } \\
\text { items }\end{array}$ & $\mathrm{X}$ \\
\hline $\begin{array}{l}\text { Barrera, Caples, and } \\
\text { Tein, } 2001\end{array}$ & $\begin{array}{l}\text { economic } \\
\text { hardship }\end{array}$ & $\begin{array}{l}\text { "Cognitive appraisal that income is } \\
\text { insufficient to provide necessities for } \\
\text { living, behaviors that reflect efforts to } \\
\text { adjust to inadequate income, and affect } \\
\text { that takes the form of despair and hopeless } \\
\text { that one's economic future will be bright" } \\
\text { (p. 495) }\end{array}$ & $\begin{array}{l}\text { Scale development: } \\
\text { three scales from } \\
\text { Conger and Elder } \\
\text { (1994), and scale } \\
\text { from Vinokur et al. } \\
\text { (1996). }\end{array}$ & $\begin{array}{l}\text { Adapted } \\
\text { from } \\
\text { previous } \\
\text { scales }\end{array}$ & $\begin{array}{l}\text { Previously } \\
\text { validated } \\
\text { scales used }\end{array}$ & $\begin{array}{l}\text { Vinokur et } \\
\text { al. (1996) } \\
\text { scale }\end{array}$ & $\begin{array}{l}\text { Conger and Elder } \\
\text { (1994) scales }\end{array}$ \\
\hline $\begin{array}{l}\text { Berg, Kiviruusu, } \\
\text { Karvonene, } \\
\text { Rahkonen, and } \\
\text { Huurre, } 2017 \\
\end{array}$ & $\begin{array}{l}\text { economic } \\
\text { adversity }\end{array}$ & $\begin{array}{l}\text { No conceptual definition provided by } \\
\text { authors }\end{array}$ & $\begin{array}{l}\text { Used three items to } \\
\text { obtain score. }\end{array}$ & Yes & Face validity & $\begin{array}{l}33 \% \text { of } \\
\text { items }\end{array}$ & $66 \%$ of items \\
\hline $\begin{array}{l}\text { Bhargava, Palmer, } \\
\text { Chatterjee, and } \\
\text { Stebbins, } 2018\end{array}$ & $\begin{array}{l}\text { financial } \\
\text { distress }\end{array}$ & $\begin{array}{l}\text { No conceptual definition provided by } \\
\text { authors }\end{array}$ & $\begin{array}{l}\text { Adapted Zagorsky } \\
2007 \text { measure as one } \\
\text { item }\end{array}$ & $\begin{array}{l}\text { Adapted } \\
\text { from } \\
\text { previous } \\
\text { scale }\end{array}$ & Face validity & $\begin{array}{l}100 \% \text { of } \\
\text { items }\end{array}$ & $\mathrm{X}$ \\
\hline $\begin{array}{l}\text { Bradshaw and } \\
\text { Ellison, } 2010\end{array}$ & $\begin{array}{l}\text { financial } \\
\text { hardship }\end{array}$ & $\begin{array}{l}\text { No conceptual definition provided by } \\
\text { authors }\end{array}$ & $\begin{array}{l}\text { Personal income and } \\
\text { perceived income. }\end{array}$ & Yes & Face validity & $\begin{array}{l}\text { Personal } \\
\text { income } \\
\text { items }\end{array}$ & $\begin{array}{l}\text { Perceived income } \\
\text { items }\end{array}$ \\
\hline $\begin{array}{l}\text { Braveman, Heck, } \\
\text { Egerter, Rinki, }\end{array}$ & $\begin{array}{l}\text { economic } \\
\text { hardship/ }\end{array}$ & $\begin{array}{l}\text { No conceptual definition provided by } \\
\text { authors }\end{array}$ & $\begin{array}{l}\text { Assessed difficulty } \\
\text { meeting basic needs }\end{array}$ & Yes & Face validity & $\begin{array}{l}100 \% \text { of } \\
\text { items }\end{array}$ & $\mathrm{X}$ \\
\hline $\begin{array}{l}\text { Marchi, and Curtis, } \\
2018\end{array}$ & $\begin{array}{l}\text { economic } \\
\text { adversity }\end{array}$ & & $\begin{array}{l}\text { (i.e., hunger and } \\
\text { rent/mortgage } \\
\text { problems) during } \\
\text { childhood; and } \\
\text { frequency of } \\
\text { difficulty. }\end{array}$ & & & & \\
\hline $\begin{array}{l}\text { Britt, Canale, Fernatt, } \\
\text { Stutz, and Tibbetts, } \\
2015\end{array}$ & financial stress & $\begin{array}{l}\text { "the inability to meet one's economic } \\
\text { responsibilities" (p. 173) }\end{array}$ & $\begin{array}{l}\text { The amount of stress } \\
\text { the respondent felt in } \\
\text { relation to their } \\
\text { personal finances }\end{array}$ & $\begin{array}{l}\text { Similar } \\
\text { questions } \\
\text { used } \\
\text { elsewhere }\end{array}$ & Face validity & $\mathrm{X}$ & All items \\
\hline $\begin{array}{l}\text { Bultena, Lasley and } \\
\text { Geller, } 1986\end{array}$ & $\begin{array}{l}\text { financial } \\
\text { distress }\end{array}$ & $\begin{array}{l}\text { No conceptual definition provided by } \\
\text { authors }\end{array}$ & $\begin{array}{l}\text { Debt to asset ratio } \\
\text { (e.g., Jolly et al., } \\
\text { 1985; Melichar, } \\
\text { 1984) }\end{array}$ & $\begin{array}{l}\text { Note similar } \\
\text { methods } \\
\text { used by } \\
\text { other } \\
\text { economists } \\
\end{array}$ & Face validity & $\begin{array}{l}100 \% \text { of } \\
\text { items }\end{array}$ & $\mathrm{X}$ \\
\hline $\begin{array}{l}\text { Del Río and Young, } \\
2005\end{array}$ & $\begin{array}{l}\text { financial } \\
\text { distress }\end{array}$ & $\begin{array}{l}\text { "The extent to which households consider } \\
\text { unsecured debt to be a burden" }\end{array}$ & $\begin{array}{l}\text { Part of the British } \\
\text { Household Panel } \\
\text { Survey (BHPS) }\end{array}$ & $\begin{array}{l}\text { Not } \\
\text { applicable }\end{array}$ & Not applicable & $\mathrm{X}$ & $100 \%$ of items \\
\hline $\begin{array}{l}\text { Glei, Goldman, and } \\
\text { Weinstein, } 2018\end{array}$ & $\begin{array}{l}\text { economic } \\
\text { distress } \\
\text { financial strain }\end{array}$ & $\begin{array}{l}\text { Reference Voydanoff (1990)'s definition } \\
\text { of economic distress as comprised of } \\
\text { objective indicators (employment } \\
\text { instability and economic deprivation) and } \\
\text { subjective indicators (financial strain and } \\
\text { unemployment uncertainty). } \\
\text { Economic deprivation was related to low } \\
\text { income and assets, whereas financial strain } \\
\text { was described as perceived financial } \\
\text { inadequacy. }\end{array}$ & $\begin{array}{l}4 \text { subjective } \\
\text { measures: five-item } \\
\text { index of current } \\
\text { financial strain, } \\
\text { intergenerational } \\
\text { financial } \\
\text { disadvantage } \\
\text { question, current and } \\
\text { expected work } \\
\text { situations. }\end{array}$ & Yes & Face Validity & $\begin{array}{l}\text { All } 4 \\
\text { subjective } \\
\text { measures }\end{array}$ & $\begin{array}{c}\text { Relative SES } \\
\text { measure }\end{array}$ \\
\hline $\begin{array}{l}\text { Grossi, Perski, } \\
\text { Lundberg, and } \\
\text { Soares, } 2001\end{array}$ & financial strain & $\begin{array}{l}\text { "Subjective experience of his/her financial } \\
\text { situation" (p. 208) }\end{array}$ & $\begin{array}{l}\text { Adapted from Pearlin } \\
\text { et al. (1981) } \\
\text { Economic Strain } \\
\text { Model; used via } \\
\text { Brenner et al. (1988) }\end{array}$ & $\begin{array}{l}\text { Adapted } \\
\text { from } \\
\text { previous } \\
\text { scale }\end{array}$ & $\begin{array}{l}\text { Not indicated } \\
\text { by Pearlin et } \\
\text { a1., } 1981\end{array}$ & $\begin{array}{l}100 \% \text { of } \\
\text { items }\end{array}$ & $\mathrm{X}$ \\
\hline $\begin{array}{l}\text { Gutman, McLoyd, } \\
\text { and Tokoyawa, } 2005\end{array}$ & financial strain & $\begin{array}{l}\text { No conceptual definition provided by } \\
\text { authors }\end{array}$ & $\begin{array}{l}\text { "(a) difficulties in } \\
\text { paying bills, (b) not } \\
\text { having enough } \\
\text { money to make ends } \\
\text { meet, and (c) } \\
\text { worrying about not }\end{array}$ & Yes & Face validity & $\begin{array}{l}100 \% \text { of } \\
\text { items }\end{array}$ & $\mathrm{X}$ \\
\hline
\end{tabular}




\begin{tabular}{|c|c|c|c|c|c|c|c|}
\hline & & & $\begin{array}{l}\text { having enough } \\
\text { money" }\end{array}$ & & & & \\
\hline $\begin{array}{l}\text { Hamid and Abdullah, } \\
2009\end{array}$ & $\begin{array}{l}\text { financial } \\
\text { distress }\end{array}$ & $\begin{array}{l}\text { "A reaction, such as mental or physical } \\
\text { discomfort, to stress about one's state of } \\
\text { general financial well being" (p. 395) }\end{array}$ & $\begin{array}{l}\text { Satisfaction on Their } \\
\text { Financial Situation } \\
\text { and Causes of } \\
\text { Financial Problems }\end{array}$ & Yes & Face validity & $\begin{array}{l}\text { causes of } \\
\text { financial } \\
\text { problems } \\
\text { scale }\end{array}$ & $\begin{array}{l}\text { satisfaction on } \\
\text { their financial } \\
\text { situation scale }\end{array}$ \\
\hline Joo, 1998 & financial stress & $\begin{array}{l}\text { "The stress that is caused by financial } \\
\text { situations including personal, family, and } \\
\text { other financial circumstances" (p.9) }\end{array}$ & $\begin{array}{l}\text { Financial Stressors } \\
\text { Scale and Financial } \\
\text { Stressor Index }\end{array}$ & Yes & Face validity. & $\begin{array}{l}100 \% \text { of } \\
\text { items }\end{array}$ & $\mathrm{X}$ \\
\hline $\begin{array}{l}\text { Kahn and Pearlin, } \\
2006\end{array}$ & financial strain & No conceptual definition provided. & $\begin{array}{l}\text { Household income. } \\
\text { Financial strain } \\
\text { measure adapted } \\
\text { from Pearlin et al. } \\
(1981) \\
\end{array}$ & No & Face validity & $\mathrm{X}$ & $100 \%$ of items \\
\hline $\begin{array}{l}\text { Kalkhoran, } \\
\text { Berkowitz, Rigotti, } \\
\text { and Baggett, } 2018\end{array}$ & $\begin{array}{l}\text { financial } \\
\text { strain/ } \\
\text { financial stress }\end{array}$ & $\begin{array}{l}\text { "Difficulty meeting financial needs and } \\
\text { obligations" (p. 81) }\end{array}$ & $\begin{array}{l}\text { Assessed whether a } \\
\text { shortage of money } \\
\text { resulted in bills not } \\
\text { being paid on time }\end{array}$ & $\begin{array}{l}\text { Reportedly, } \\
\text { similar to } \\
\text { other } \\
\text { measures }\end{array}$ & Face validity & $\begin{array}{l}100 \% \text { of } \\
\text { item }\end{array}$ & $\mathrm{X}$ \\
\hline $\begin{array}{l}\text { Kiely, Leach, Olesen } \\
\text { and Butterworth, } \\
2015\end{array}$ & $\begin{array}{l}\text { financial } \\
\text { hardship }\end{array}$ & $\begin{array}{l}\text { "Differentiate between two aspects of } \\
\text { financial hardship: cash flow problems and } \\
\text { deprivation. Specifically, cash-flow } \\
\text { problems are relatively commonly reported } \\
\text { and define circumstances in which regular } \\
\text { bills may not be paid on time, or occasions } \\
\text { when financial assistance is sought from } \\
\text { family members ... deprivation refers to } \\
\text { less common events whereby people are } \\
\text { unable to provide the essentials of life and } \\
\text { cannot meet the basic standards of living } \\
\text { accepted in society" (p. } 910)\end{array}$ & $\begin{array}{l}\text { Measure of financial } \\
\text { hardship adapted } \\
\text { from Bray }(2001) \text { and } \\
\text { Butterworth and } \\
\text { Crosier (2006) }\end{array}$ & No & $\begin{array}{l}\text { Criterion } \\
\text { validity } \\
\text { previously } \\
\text { established }\end{array}$ & $\begin{array}{l}100 \% \text { of } \\
\text { items }\end{array}$ & $\mathrm{X}$ \\
\hline $\begin{array}{l}\text { Kim and Garman, } \\
2003\end{array}$ & $\begin{array}{l}\text { financial } \\
\text { stress/ } \\
\text { economic } \\
\text { stress }\end{array}$ & $\begin{array}{l}\text { "Subjective perception of one's personal } \\
\text { finances" (p. 35) }\end{array}$ & $\begin{array}{l}\text { Adapted financial } \\
\text { stress scales used in } \\
\text { Joo (1998) and } \\
\text { Bagwell (2000) }\end{array}$ & No & Face validity & $\mathrm{X}$ & $100 \%$ of items \\
\hline \multirow[t]{2}{*}{$\begin{array}{l}\text { Latsou and Geitona, } \\
2018\end{array}$} & $\begin{array}{l}\text { economic } \\
\text { distress }\end{array}$ & $\begin{array}{l}\text { No conceptual definition provided by } \\
\text { authors }\end{array}$ & $\begin{array}{l}\text { Index of personal } \\
\text { economic distress } \\
\text { (IPED; Madianos, } \\
\text { Economou, Alexiou, } \\
\text { and Stefanis, 2011) } \\
\end{array}$ & No & $\begin{array}{l}\text { Content } \\
\text { validity } \\
\text { measured } \\
\text { against } \\
\text { socioconomic } \\
\end{array}$ & $\begin{array}{l}\text { roughly } \\
62.5 \% \text { of } \\
\text { items }\end{array}$ & $\begin{array}{l}\text { roughly } 37.5 \% \text { of } \\
\text { items }\end{array}$ \\
\hline & & & & & status (SES) & & \\
\hline $\begin{array}{l}\text { Lim, Heckman, } \\
\text { Letkiewicz, and } \\
\text { Montalto, } 2014\end{array}$ & financial stress & $\begin{array}{l}\text { No conceptual definition provided by } \\
\text { authors }\end{array}$ & $\begin{array}{l}\text { Used six items to } \\
\text { obtain scores }\end{array}$ & Yes & Face validity & $\mathrm{X}$ & $100 \%$ of items \\
\hline McCarthy, 2011 & $\begin{array}{l}\text { financial } \\
\text { distress/ } \\
\text { financial } \\
\text { difficulty }\end{array}$ & $\begin{array}{l}\text { "A situation where individuals report that } \\
\text { they are having some degree of difficulty } \\
\text { keeping up with their bills and credit } \\
\text { commitments" (p. 11) }\end{array}$ & $\begin{array}{l}\text { Financial Capability } \\
\text { Survey }\end{array}$ & No & $\begin{array}{l}\text { Pilot studies } \\
\text { conducted, but } \\
\text { no scale } \\
\text { validation }\end{array}$ & $\begin{array}{l}100 \% \text { of } \\
\text { items }\end{array}$ & $\mathrm{X}$ \\
\hline $\begin{array}{l}\text { Mills, Grasmick, } \\
\text { Morgan, and Wenk, } \\
1992\end{array}$ & $\begin{array}{l}\text { economic } \\
\text { strain }\end{array}$ & $\begin{array}{l}\text { Voydanoff's (1990) definition as "an } \\
\text { individual's perception of financial } \\
\text { inadequacy as well as her/his concerns and } \\
\text { worries" (p. 440) }\end{array}$ & $\begin{array}{l}\text { Used four items to } \\
\text { obtain score. }\end{array}$ & Yes & Face validity & $\mathrm{X}$ & $100 \%$ of items \\
\hline $\begin{array}{l}\text { ONeill, Xiao, } \\
\text { Sorhaindo, and } \\
\text { Garman, } 2005\end{array}$ & $\begin{array}{l}\text { financial } \\
\text { stress/ } \\
\text { negative } \\
\text { financial } \\
\text { events }\end{array}$ & $\begin{array}{l}\text { No conceptual definition provided by } \\
\text { authors }\end{array}$ & $\begin{array}{l}\text { Used one item to } \\
\text { obtain a financial } \\
\text { stress score, and } 12 \\
\text { items to obtain a } \\
\text { negative financial } \\
\text { event score }\end{array}$ & Yes & Face validity & $\begin{array}{l}\text { negative } \\
\text { financial } \\
\text { items }\end{array}$ & $\begin{array}{l}\text { financial stress } \\
\text { items }\end{array}$ \\
\hline $\begin{array}{l}\text { Park, Heo, Ruiz- } \\
\text { Menjivar, and Grable, } \\
2017\end{array}$ & $\begin{array}{l}\text { financial } \\
\text { hardship }\end{array}$ & $\begin{array}{l}\text { "An individual's disadvantageous financial } \\
\text { condition" (p. 323) }\end{array}$ & $\begin{array}{l}\text { Used three items to } \\
\text { obtain score. }\end{array}$ & Yes & Face validity & $\begin{array}{l}100 \% \text { of } \\
\text { items }\end{array}$ & $\mathrm{X}$ \\
\hline $\begin{array}{l}\text { Pearlin, Lieberman, } \\
\text { Menaghan, and } \\
\text { Mullin, } 1981\end{array}$ & $\begin{array}{l}\text { economic } \\
\text { strain }\end{array}$ & $\begin{array}{l}\text { Difficulties meeting basic needs, and } \\
\text { difficulties acquiring non-necessary, but } \\
\text { desired items. }\end{array}$ & $\begin{array}{l}\text { Economic strain } \\
\text { model }\end{array}$ & Yes & Face validity & $\mathrm{X}$ & $100 \%$ of items \\
\hline $\begin{array}{l}\text { Perry, Hoerger, } \\
\text { Seibert, Gerhart, } \\
\text { O'Mahony, and } \\
\text { Duberstein, } 2019 \\
\end{array}$ & financial strain & $\begin{array}{l}\text { "Lacking the financial resources necessary } \\
\text { to meet basic needs" (p. 454) }\end{array}$ & $\begin{array}{l}\text { Scale developed by } \\
\text { Friedman, Conwell, } \\
\text { and Delavan (2007) }\end{array}$ & No & Face validity & $\mathrm{X}$ & $100 \%$ of items \\
\hline Perry-Rivers, 2014 & $\begin{array}{l}\text { economic } \\
\text { adversity }\end{array}$ & $\begin{array}{l}\text { No conceptual definition provided by } \\
\text { authors }\end{array}$ & $\begin{array}{l}\text { Coded as recession } \\
\text { and non-recession } \\
\text { years }\end{array}$ & No & Face validity & $\mathrm{X}$ & $\begin{array}{l}\text { Single recession } \\
\text { item }\end{array}$ \\
\hline Prawitz et al., 2006 & $\begin{array}{l}\text { financial } \\
\text { distress }\end{array}$ & $\begin{array}{l}\text { "The construct encompassing perceptions } \\
\text { about financial well-being and stress about } \\
\text { one's financial condition" (p. 36) }\end{array}$ & IFDFW & Yes & $\begin{array}{l}\text { Established } \\
\text { construct and } \\
\text { criterion } \\
\text { validity }\end{array}$ & $\mathrm{X}$ & $100 \%$ of items \\
\hline $\begin{array}{l}\text { Price, Choi, and } \\
\text { Vinokur, } 2002\end{array}$ & financial strain & $\begin{array}{l}\text { No conceptual definition provided by } \\
\text { authors }\end{array}$ & $\begin{array}{l}\text { Scale developed by } \\
\text { Vinokur and Caplan } \\
\text { (1987) }\end{array}$ & No & Face validity & $\mathrm{x}$ & $100 \%$ of items \\
\hline
\end{tabular}




\begin{tabular}{|c|c|c|c|c|c|c|c|}
\hline $\begin{array}{l}\text { Starrin, Aslund, } \\
\text { Nilsson, } 2009\end{array}$ & $\begin{array}{l}\text { financial } \\
\text { stress/ } \\
\text { hardship/ } \\
\text { strain/ } \\
\text { vulnerability/ } \\
\text { deprivation } \\
\end{array}$ & $\begin{array}{l}\text { "Problems that arise when a person is } \\
\text { faced with the threat of a shortage of } \\
\text { money in combination with a lack of cash } \\
\text { reserves ad difficulties making ends meet" } \\
\text { (p. 285) }\end{array}$ & $\begin{array}{l}\text { Finances-Shame } \\
\text { Model }\end{array}$ & Yes & Face validity & $\begin{array}{l}\text { financial } \\
\text { stress items }\end{array}$ & $\begin{array}{l}\text { financial shame } \\
\text { items }\end{array}$ \\
\hline Tormos, 2019 & $\begin{array}{l}\text { economic } \\
\text { hardship }\end{array}$ & $\begin{array}{l}\text { No conceptual definition provided by } \\
\text { authors }\end{array}$ & $\begin{array}{l}\text { Used five items to } \\
\text { assess conditions, } \\
\text { consequences, and } \\
\text { distress caused by } \\
\text { economic hardship }\end{array}$ & Yes & Face validity & $\begin{array}{l}100 \% \text { of } \\
\text { items }\end{array}$ & $\mathrm{X}$ \\
\hline $\begin{array}{l}\text { Vinokur and Caplan, } \\
1987\end{array}$ & $\begin{array}{l}\text { economic } \\
\text { hardship }\end{array}$ & $\begin{array}{l}\text { No conceptual definition provided by } \\
\text { authors }\end{array}$ & $\begin{array}{l}\text { Used three items to } \\
\text { obtain score. }\end{array}$ & Yes & Face validity & $\mathrm{X}$ & $100 \%$ of items \\
\hline $\begin{array}{l}\text { Viseu, Leal, Neves de } \\
\text { Jesus, Pinto, } \\
\text { Pechorro, and } \\
\text { Greenglass, } 2018\end{array}$ & $\begin{array}{l}\text { economic } \\
\text { hardship/ } \\
\text { economic } \\
\text { stress factors }\end{array}$ & $\begin{array}{l}\text { Economic stress factors described as } \\
\text { economic hardship, financial threat, and } \\
\text { financial well-being. No further conceptual } \\
\text { definitions provided by authors. }\end{array}$ & $\begin{array}{l}\text { Economic hardship } \\
\text { questionnaire (EHQ; } \\
\text { Leal et al., 2014) and } \\
\text { financial threat scale } \\
\text { (FTS; Marjanovic et } \\
\text { al., 2013). }\end{array}$ & No & & $\mathrm{X}$ & $100 \%$ of items \\
\hline Wharton, 2007 & financial stress & $\begin{array}{l}\text { No conceptual definition provided by } \\
\text { author }\end{array}$ & $\begin{array}{l}\text { Financial Wellness } \\
\text { Survey }\end{array}$ & Yes & $\begin{array}{l}\text { Criterion } \\
\text { validity } \\
\text { measured with } \\
\text { PCA }\end{array}$ & $\begin{array}{l}\text { roughly } 65 \% \\
\text { of items }\end{array}$ & $\begin{array}{l}\text { roughly } 35 \% \text { of } \\
\text { items }\end{array}$ \\
\hline Zagorsky, 2007 & $\begin{array}{l}\text { financial } \\
\text { distress }\end{array}$ & "Living beyond one's means" (p. 492) & $\begin{array}{l}\text { Used three items to } \\
\text { obtain score. }\end{array}$ & Yes & Face validity & $\begin{array}{l}100 \% \text { of } \\
\text { items }\end{array}$ & $\mathrm{X}$ \\
\hline $\begin{array}{l}\text { Zheng, } \mathrm{Xu}, \mathrm{Xu} \text {, } \\
\text { Yang, Gu, and Wang, } \\
2019\end{array}$ & $\begin{array}{l}\text { financial } \\
\text { strain/ } \\
\text { financial } \\
\text { difficulties/ } \\
\text { economic } \\
\text { hardship }\end{array}$ & $\begin{array}{l}\text { Defined financial strain as "lack of } \\
\text { financial resources to meet family needs, } \\
\text { due to low income, job loss, temporary or } \\
\text { chronic unemployment" (p. 5, cited Glei, } \\
\text { Goldman, and Weinstein, 2018). } \\
\text { Defined perceived financial strain as "self- } \\
\text { assessed or perceived financial difficulties } \\
\text { and unemployment circumstances" (p. 7, } \\
\text { cited Glei et al., 2018). }\end{array}$ & $\begin{array}{l}\text { Perceived financial } \\
\text { strain assessed } \\
\text { through seven items } \\
\text { about lack of funds } \\
\text { and high cost of } \\
\text { living. } \\
\text { Financial strain } \\
\text { assessed with two } \\
\text { items about income } \\
\text { and unemployment. }\end{array}$ & Yes & Face validity & $\begin{array}{l}\text { Financial } \\
\text { strain items }\end{array}$ & $\begin{array}{c}\text { Perceived } \\
\text { financial strain } \\
\text { items }\end{array}$ \\
\hline
\end{tabular}

Economic hardship, for example, was discussed in 5 studies out of 37, but only one provided a definition as follows: "Cognitive appraisal that income is insufficient to provide necessities for living, behaviors that reflect efforts to adjust to inadequate income, and affect that takes the form of despair and hopeless that one's economic future will be bright" (Barrera, Caples, \& Tein, 2001). Financial distress, was discussed in 8 studies with 7 of these providing the following definitions: "Situations in which the household remains susceptible to any deviation of income from its ex-ante expectation" (Athreya et a., 2017); "The extent to which households consider unsecured debt to be a burden" (Del Río \& Young, 2005); "A reaction, such as mental or physical discomfort, to stress about one's state of general financial well being" (Hamid \& Abdullah, 2009); "A situation where individuals report that they are having some degree of difficulty keeping up with their bills and credit commitments" (McCarthy, 2011), etc. Economic deprivation was not defined in any of the 37 selected articles.

Financial strain was discussed in 11 studies out of 37 and definitions were provided in 7 studies out of 11 . Definitions ranged from "Composed of the cognitive, emotional, and behavioral response to the experience of financial hardship" (Aldana \& Liljenquist 1998); "Subjective experience of his/her financial situation" (Grossi, Perski, Lundberg, \& Soares, 2001); "perceived financial inadequacy" (Glei, Goldman, and Weinstein, 2018); "Difficulty meeting financial needs and obligations" (Kalkhoran, Berkowitz, Rigotti, \& Bagget,t 2018); "Lacking the financial resources necessary to meet basic needs" (Perry et al., 2019); and "self-assessed or perceived financial difficulties and unemployment circumstances" (Zheng et al., 2019).

Economic strain was mentioned in two studies and both provided definitions as follows: "Difficulties meeting basic needs, and difficulties acquiring non-necessary, but desired items" (Pearlin, Lieberman, Menaghan, \& Mullin, 1981) and "an individual's perception of financial inadequacy as well as her/his concerns and worries" (Mills et al., 1992 citing Voydanoff, 1990).

\subsection{Scales}

We observed a large variety of scales used in our sample. For example, two studies developed new scales for economic hardship based on previous scales (Barrera et al., 2001; Vinokur \& Caplan, 1987). Another assessed difficulty meeting basic needs (i.e., hunger and rent/mortgage problems) during childhood and frequency of difficulty (Braveman et al., 2018); another used five items to assess conditions, consequences, and distress caused by economic hardship (Tormos 2019), and yet another used the economic hardship questionnaire (EHQ; by Leal et al., 2014) (Viseu et al., 2018). For financial distress, studies used Equifax data (no scale) (Athreya et al., 2017); one study used measures adapted from a previous scale (Bhargava et al., 2018; Zagorsky, 2007); one used debt to asset ratio (Bultena, Lasley, \& Geller, 1986); and several used various scales such as the Part of the British Household Panel Survey (BHPS) (Del Río \& Young, 2005), Satisfaction on Their Financial Situation and 
Causes of Financial Problems (Hamid \& Abdullah, 2009), or the Financial Capability Survey (McCarthy, 2011). In total, 20 new scales were developed, whereas seven were measures adapted from previous studies.

\subsection{Validation of Assessment Tools for Personal Negative Financial Health}

Most self-reporting measures of personal negative financial health demonstrate face validity: scales appear to measure the latent negative financial construct they are being used to measure (Kaplan \& Saccuzzo, 2017). However, face validity does not empirically support assertions that a scale is useful or meaningful to the underlying construct. Of the 37 papers included for analysis of their conceptual and operational definitions, only six demonstrated an additional validity test (see Table 3; i.e., Aldana \& Lijenquist, 1998; Barrera et al, 2001; Kiely, Leach, Olesen, \& Butterworth, 2015; Latsou \& Geitona, 2018; Prawitz et al., 2006; Wharton, 2007). Of those studies, four provided empirical evidence of validity, through construct and/or criterion validity. One study indicated that the assessment tool was previously validated, and the final study provided supplementary non-empirical evidence of validity by correlating measures scores with socioeconomic status (Latsou \& Geitona, 2018). Although discriminant validity is heralded as the best way to prevent construct proliferation (Shaffer, DeGeest, \& $\mathrm{Li}, 2016$ ), none of the articles that tested validity reported discriminant validity.

\subsection{Assessing and Conceptualizing Personal Negative Financial Health Terms}

In the following, objective and subjective measures are further reviewed, with an emphasis on how negative financial health terms have been objectively and subjectively measured and validated.

\subsubsection{Objective Measures}

Objective measures of financial distress describe financial behaviors wherein people have difficulty maintaining their financial obligations, as evidenced by an accumulation of credit arrears or running out of money (McCarthy, 2011). Financial stress has similarly been conceptualized as one's chronic inability to maintain a basic standard of living (Åslund, Larm, Starrin, \& Nilsson, 2014), or problems arising from one's lack of funds to meet financial obligations and their failure to save additional funds (Starrin, Åslund, \& Nilsson, 2009). Starrin et al. (2009) operationalized financial stress as one's frequency of difficulty in meeting monthly financial obligations during the past three months; one's frequency of raising additional funds through (a) borrowing money, (b) pawning items, (c) selling items, or (d) applying for welfare during the past three months; one's frequency of limiting or refraining from extraneous purchases of (a) food, (b) clothing, or (c) vacations; and one's inability to accrue additional emergency funds. Financial stress has been similarly operationalized by participants indicating which personal stressors (e.g., job change, wage decrease, injury), family events (e.g., divorce, marriage, birth of a child), and financial stress circumstances (e.g., house foreclosure, vehicle repossession, excessive consumer debt) contributed to their experience of financial stress (Wharton, 2007). Debt-to asset ratios may also be used to operationalize financial distress (Bultena et al., 1986). For example, someone who experiences cash flow problems, or consistently has greater debts than assets, would be more likely to experience financial distress. Through comparison of objective measures and the conceptual definitions provided for financial distress and financial stress, it is evident that these terms have been similarly described in scholarly literature.

Objective measures typically favor the terminology financial distress and financial stress when describing negative financial behaviors; however, financial hardship and financial strain have also been used as substitutes. For example, financial hardship may be indicated by unemployment, cutting back expenses, being without meals, and asking for financial help (reviewed in Park et al., 2017). Economic hardship may also be operationalized through high debt-to-asset ratios, large reductions in family income, or income below the poverty line (Davis \& Mantler, 2004). Although financial strain and financial hardship are defined analogously to financial stress and distress, they are more commonly assessed through subjective assessments rather than objective measures (e.g., Aldana \& Liljenquist, 1998; Gutman, McLoyd, \& Tokoyawa, 2005; Vinokur \& Caplan, 1987).

\subsubsection{Subjective Assessments}

When referring to negative financial health behaviors, subjective measures are used more frequently than objective measures. The predisposition to use subjective assessments stems from a greater ability to gauge perceived financial needs, and the standardization of subjective measures (Barrera et al., 2001). Subjective assessments typically recognize that two people may experience different reactions to potential financial stressors; therefore, it may be more important to ask participants about the amount of distress they felt than the quantity of possible financial stressors they experienced. Subjective measures have also been favored because they address whether financial stress arises from a disparity between one's financial needs and their financial resources (Barrera et al., 2001).

Subjective assessments inquire about experiences of perceived financial stress. For example, financial distress is 
operationalized as the magnitude of burden, or the amount of distress one experiences because of their unsecured debt (Del-Río \& Young, 2005). Moreover, since diverse financial conditions only produce financial distress under certain circumstances (Joo, 1998), it is possible for certain negative financial situations may not produce financial distress for certain people. Different opinions also exist on whether financial distress arises after long-term exposure to financial stressors (Prawitz et al., 2006) and whether it can be experienced both briefly or persistently (O'Neill et al., 2006). Depending on the circumstances encountered, and measurement items used, it is possible for someone to objectively appear to be financially distressed in the absence of subjective financial distress. Similarly, it is possible for someone to be subjectively financially distressed while not objectively appearing to be financially distressed.

While objective definitions of financial hardship and financial strain are analogous with financial distress, subjective operationalizations appear more disparate. Most definitions of financial distress and financial stress focus on the present and present circumstances, financial hardship and financial strain may refer to more transient experiences that are currently experienced, or may be experienced in the future (e.g., Hamid \& Abdullah, 2009; O'Neill et al., 2006; Price, Choi, \& Vinokur 2002). Financial strain has been conceptualized as an inability to manage income and maintain savings, pay bills, repay debt, and have excess finances to cover desired expenditures (Hamid \& Abdullah, 2009; O'Neill et al., 2006). Financial strain has also been operationalized as one's perception of current and future difficulties in using personal income to meet financial responsibilities (Price et al., 2002). However, aside from temporal differences between definitions of financial distress and financial strain, differences in the subjective assessments of financial distress terminology appear negligible.

\subsubsection{Objective and Subjective Assessments}

The combination of both objective and subjective assessments should result in a more comprehensive understanding of financial distress, than either assessment type provides alone. For example, Åslund and colleagues (2014) reduced and modified Starrin and colleagues' (2009) operationalization of financial stress. The new conceptualization referred to financial stress as the degree of difficulty in meeting monthly financial obligations during the previous year, and an inability to accrue additional emergency funds. Financial distress was also operationalized as perceptions of current and previous experiences of financial stress, and whether respondents had a history of bankruptcy (Zagorsky, 2007). Similarly, financial strain was operationalized as the inability to afford a suitable home, furnishings, car, food, medical care, clothing, and recreational activities; the degree of difficulty in meeting financial obligations; and the financial resources available after paying monthly financial expenses (Grossi et al., 2001). These examples of combined objective and subjective assessments analyze objective factors of how often something occurred, in conjunction with the perceived extent or degree of difficulty the individual felt.

Other conceptualizations of financial distress describe objective assessments of income or debts, and perceptions of income deprivation or worry. For example, one study utilized the lowest personal income quartile in conjunction with assessments of perceived income deprivation (Bradshaw \& Ellison, 2010). Another study defined financial hardship as one's perceived difficulty paying monthly bills, the amount of debt they accrued when trying to pay monthly bills, and whether they received government assistance (Park et al., 2017). Financial hardship was also conceptualized as the combination of cash flow deficits and perceptions of financial deprivation (Kiely et al., 2015). Thus, the combination of objective and subjective measures enables researchers to differentiate between groups of people with different levels of financial resources and different levels of severity (e.g., Kiely et al., 2015).

\section{Discussion}

Financial behaviors are studied by disparate disciplines, posing potential problems and benefits to academics and practitioners. Collaboration and integration between academic disciplines and practitioners has been prompted by the need for practical problem-solving (Hadorn, Pohl, \& Bammer, 2010). Given that different perspectives increase understanding and resolution of complex problems, interdisciplinary and transdisciplinary research are increasingly popularized as solutions to integration problems (Klein, 1990; 1996; 2010). The largest obstacle to integrated research is construct proliferation (Klein, 1990; 1996). Although multidisciplinarity juxtaposes theoretical and conceptual practices between disciplines, it does not prescribe a course of best action (Klein, 2010). Moreover, because of the disparity between negative financial health terminology, integration is needed to clarify and unify existing concepts. We recommend the following for researchers, practitioners, and individuals conducting transdisciplinary work.

Recommendation 1. Where possible, authors should provide consistent conceptual and operational definitions of 
negative financial health constructs. They should seek to identify and clearly communicate relevant synonyms in their research, especially when researchers prefer a negative financial health term that differs from the term used in their operational definition. Merely providing information on the operationalization of the term or the measure that researchers will use is insufficient for communicating their understanding of the term (e.g., Dumont \& Wilson, 1967; Hempel, 1965; Lester \& Lester Jr., 2015).

Recommendation 2. Researchers should provide synonymous terms as keywords when publishing research on negative financial constructs. Evidence in biological studies suggests that the use of synonymous terms, such as an organism's scientific and common names, substantially increase the yield of extant scientific information (e.g., Guala, 2016). In their study, Guala (2016) found the yield substantially increased with the inclusion of one additional synonym. Based on their prevalence across databases, we recommend including economic hardship and financial strain as keywords. Researchers may also choose to utilize related discipline-specific keywords instead.

Recommendation 3. Researchers developing new measures of negative financial health constructs should assess and report discriminant validity. Scale validity is an important aspect of communicating how a measurement instrument accurately assesses financial health (Kimberlin \& Winterstein, 2008). Reporting measures of validity, especially discriminant validity, serve to increase scientific rigour and reduce construct proliferation (Kimberlin \& Winterstein, 2008; Shaffer et al., 2016). Scale developers should strive for and report more rigorous indicators of validity than face validity. Moreover, when researchers use measurement instruments to study new populations, scale validation should be replicated to ensure the scale is a valid measure of negative financial health within the new population.

Recommendation 4. Researchers, practitioners, and knowledge-mobilizers should communicate information about negative financial health to the public using financial hardship, financial stress, or financial distress. In order to better communicate with the general public, researchers and practitioners should utilize the search terms that members of the public prefer. Within the last five years, an increasing proportion of the global population has searched for information using financial hardship, financial stress, and/or financial distress (Google, 2021). In order to increase the likelihood of connecting with the public in online publications-including open-source journal articles - and social marketing campaigns, researchers, practitioners, and knowledge-mobilizers need to speak the same language and use the same terms that people are engaging with. Based on geographic region, different negative financial health terms would be required to best communicate with the target audience. Practitioners and knowledge-mobilizers should also consider reviewing real time Google Trends data for the relative interest in search terms within their specific target region, as there may be slight regional variations. When optimizing Google ads, practitioners should also consider using these preferred terms to reach a larger audience.

Recommendation 5. Researchers should consider utilizing both objective and subjective indicators of negative financial health. Analysis of negative financial health terminology supports the use of both objective and subjective assessments of personal negative financial health. Utilizing both objective and subjective definitions and measurements affords unique information that is not captured by a one-dimensional conceptualization of negative financial health. It also may enable better understanding of how people perceive the financial health of those around them, in comparison with their own financial health. Through better understanding of the mechanisms which lead to divergent negative financial health assessments and determining whether and how to reconcile maladaptive assessments of negative financial health, it is possible to thoroughly comprehend the subjective nature of the financial problems people are facing, create financial well-being programs that are more equipped to meet their financial needs, and empirically evaluate the success of such programs.

By reducing or excluding objective measures, it is impossible to segment people and provide specific recommendations based on one's financial situations and how they feel about their financial situation (e.g., Cismaru \& Wuth, 2019). This makes it difficult to provide useful knowledge to community partners interested in creating interventions and programming to reduce financial distress. However, since people can experience financial well-being one moment, and poor financial health the next, it is fundamental to understand subjective experiences of negative financial health. People can experience negative financial health even at the highest income levels (e.g., Camerer, 1998), but how they experience negative financial health and the strategies they use to try and improve their financial health may differ based on individual differences. Therefore, when the scope of a study is directly related to the lived experience of negative financial health, researchers should attempt to utilize a combination of objective and subjective indicators of financial distress, and focus on the differential experiences that observe when none, one, or both indicators are present. If it is unfeasible to include both objective and subjective measures, researchers should favor subjective measures of financial distress, which 
would capture one's perception of their financial situation rather than just the resources that they do or do not have. The combination of objective and subjective indicators of financial distress will enable various community partners to determine effective interventions and treatments.

\section{Limitations and Future Research Directions}

Taken together, extant literature and big data suggest many different terms are ascribed to individual experiences of negative financial health. The terms preferred by academics and the public differ substantially, which poses problems for knowledge mobilization. Review of the use of academic terms also suggest that there were historical differences between select negative financial health constructs but these differences have disappeared over time. The results of this study have direct implications for how researchers and practitioners relay information to the general public. The results also suggest discriminant validity is required to determine whether inter-related negative financial health constructs differ substantially at an empirical level and to quantify the extent to which each construct is empirically unique. We look forward to future work that continues to bridge the gap between negative financial health knowledge development and application. Indeed, the present paper has several limitations. Only a limited number of databases were searched and only 14 negative financial health terms were considered. Searches were time-limited to 2019. Further studies could expand the analysis to include other databases such as ABI/INFORM Collection, Academic Search Complete, ArticleFirst and Emerald Fulltext, resulting in new keywords and newer articles. Further analysis of Google search data might also be of interest. For example, when customers purchase ads via Google, they have access to increased search data that shows figures of actual searches conducted for a specific term. Applying this to the success of campaigns to determine whether social marketing campaigns that utilize preferred financial terms are received better than social marketing campaigns that use less common terms could help optimize the information.

\section{Acknowledgements}

As Conexus Research Scholar in Financial Wellbeing, Magdalena Cismaru receives funds from Conexus Credit Union to conduct research in financial wellbeing.

\section{References}

Aldana, S. G., \& Liljenquist, W. (1998). Validity and reliability of a financial strain survey. Journal of Financial Counseling and Planning, 9(2), 11-19.

American Psychological Association. [APA] (2017). Stress in America ${ }^{T M}$ Press Room. Retrieved June 7, 2018, from http://www.apa.org/news/press/releases/stress/index.aspx

Åslund, C., Larm, P., Starrin, B., \& Nilsson, K. W. (2014). The buffering effect of tangible social support on financial stress: influence on psychological well-being and psychosomatic symptoms in a large sample of the adult general population. International Journal for Equity in Health, 13(85), 1-9. https://doi.org/10.1186/s12939-014-0085-3

Athreya, K., Mustre-del-Río, J., \& Sánchez, J. (2017). The persistence of financial distress. (Federal Reserve Bank [FRB] Working Paper No. 17-14). FRB Richmond, Virginia. https://doi.org/10.21144/wp17-14

Australian Psychological Society. [APS] (2015). Stress and well-being: How Australians are coping with life. Retrieved June 7, 2018, from

https://www.headsup.org.au/docs/default-source/default-document-library/stress-and-well-being-in-australia -report.pdf?sfvrsn=7f08274d_4

Bagwell, D. C. (2000). Work and personal financial outcomes of credit counseling clients (Unpublished doctoral dissertation). Virginia, Polytechnic Institute and State University, Blacksburg.

Barrera, M., Caples, H., \& Tein, J. Y. (2001). The psychological sense of economic hardship: Measurement models, validity, and cross-ethnic equivalence for urban families. American Journal of Community Psychology, 29(3), 493-517. https://doi.org/10.1023/A:101032811511

Berg, N., Kiviruusu, O., Karvonen, S., Rahkonen, O., \& Huurre, T. (2017). Pathways from poor family relationships in adolescence to economic adversity in mid-adulthood. Advances in Life Course Research, 32, 65-78. https://doi.org/10.1016/j.alcr.2016.07.001

Bernard, H. R. (2012). Social research methods: Qualitative and quantitative approaches. Sage

Bhargava, V., Palmer, L., Chatterjee, S., \& Stebbins, R. (2018). Supportive and mitigating factors associated with financial resiliency and distress. Financial Planning Review, 1e1023. https://doi.org/10.1002/cfp2.1023

Bradshaw, M., \& Ellison, C. G. (2010). Financial hardship and psychological distress: Exploring the buffering 
effects of religion. Social Science and Medicine, 71(1), 196-204.

https://doi.org/10.1016/j.socscimed.2010.03.015

Braveman, P., Heck, K., Egerter, S., Rinki, C., Marchi, K., \& Curtis, M. (2018). Economic hardship in childhood: A neglected issue in ACE studies? Maternal Child Health Journal 22, 308-317. https://doi.org/10.1007/s10995-017-2368-y

Bray, J. R. (2001). Hardship in Australia: An analysis of financial stress indicators in the 1998-1999 Australian bureau of statistics household expenditure survey. FAHCSIA, Canberra, Australia.

Britt, S. L., Canale, A., Fernatt, F., Stutz, K., \& Tibbetts, R (2015). Financial stress and financial counseling: Helping college students. Journal of Financial Counseling and Planning 26(2), 172-186. https://doi.org/10.1891/1052-3073.26.2.172

Brüggen, E. C., Hogreve, J., Holmlund, M., Kabadayi, S., \& Löfgren, M. (2017). Financial well-being: A conceptualization and research agenda. Journal of Business Research 79, 228-237. https://doi.org/10.1016/j.jbusres.2017.03.013

Bultena, G., Lasley, P., \& Geller, J. (1986). The farm crisis: Patterns and impacts of financial distress among Iowa farm families. Rural Sociology 51, 436-448.

Butterworth, P., \& Crosier, T. (2006). Deriving a measure of financial hardship from the HILDA Survey. Australian Social Policy 2005, 1 December.

Camerer, C. F. (1998). Prospect theory in the wild: Evidence from the field. (Working paper No. 1037). California Institute of Technology, Pasadena, California.

Cismaru, M., \& Wuth, A. (2019). Identifying and analyzing social marketing initiatives using a theory-based approach. Journal of Social Marketing 9(4), 357-397. https://doi.org/10.1108/JSOCM-06-2018-0063

Conger, R. D., \& Elder, G. H., Jr. (1994). Families in troubled times: Adapting to change in rural America. New York: Aldine de Gruyter.

Consumer Financial Protection Bureau. [CFPB] (2015). Financial well-being: The goal of financial education. Consumer Financial Protection Bureau, 1-48.

Retrieved from https://www.consumerfinance.gov/data-research/research-reports/financial-well-being/

Davis, C. G., \& Mantler, J. (2004). The consequences of financial stress for individuals, families, and society. Ottawa, ON: Doyle Salewski Inc.

Del-Río, A., \& Young, G. (2005). The impact of unsecured debt on financial distress among British households. (Bank of England No. 262) London: Bank of England. https://doi.org/10.2139/ssrn.824147

Dumont, R. G., \& Wilson, W. J. (1967). Aspects of concept formation, explication, and theory construction in sociology. American Sociological Review, 985-995. https://doi.org/10.2307/2092850

Friedman, B., Conwell, Y., \& Delavan, R. L. (2007). Correlates of late-life major depression: A comparison of urban and rural primary care patients. The American Journal of Geriatric Psychiatry, 15(1), 28-41. https://doi.org/10.1097/01.JGP.0000224732.74767.ad

Gallup Inc. (2018a). Gallup Global Financial Health Study. A 10-Country Survey to Measure Financial Control and Security. Retrieved from https://news.gallup.com/reports/233399/gallup-global-financial-health-study-2018.aspx

Gallup Inc. (2018b). Global Financial Health Study: A 10-Country Survey to Measure Financial Security and Control. Technical Methodology Report. Retrieved from

https://news.gallup.com/reports/233399/gallup-global-financial-health-study-2018.aspx

Glei, D. A., Goldman, N., \& Weinstein, M. (2018). Perception has its own reality: Subjective versus objective measures of economic distress. Population and development review 44(4), 695-722. https://doi.org/10.1111/padr.12183

Google. (2021, January 14). Google trends compare. Retrieved January 7, 2021, from https://www.google.com/trends

Grossi, G., Perski, A., Lundberg, U., \& Soares, J. (2001). Associations between financial strain and the diurnal salivary cortisol secretion of long-term unemployed individuals. Integrative Physiological and Behavioral Science, 36(3), 205-219. https://doi.org/10.1007/BF02734094

Guala, G. F. (2016). The importance of species name synonyms in literature searches. PLoS ONE, 11(9), 
e0162648. https://doi.org/10.1371/journal.pone.0162648

Gutman, L. M., McLoyd, V. C., \& Tokoyawa, T. (2005). Financial strain, neighborhood stress, parenting behaviors, and adolescent adjustment in urban African American families. Journal of Research on Adolescence, 15(4), 425-449. https://doi.org/10.111/j.1532-7795.2005.00106.x

Hadorn, G. H., Pohl, C., \& Bammer, G. (2010). Solving problems through transdisciplinary research. In R. Frodeman, J. T. Klein, \& C. Mitcham (Eds.), The Oxford Handbook of Interdisciplinarity. New York, NY: Oxford University Press Inc.

Hamid, N. H. A., \& Abdullah, A. (2009). Financial distress among employees: An exploratory study. Prosiding Perkem, 4(2), 395-405.

Hempel, C. G. (1965). Fundamentals of taxonomy. In C. G. Hempel (Ed.), Aspects of Scientific Explanation and Other Essays in the Philosophy of Science. New York, NY: The Free Press.

Joo, S. (1998). Personal financial wellness and worker job productivity (Unpublished doctoral dissertation). Virginia Polytechnic Institute and State University, Blacksburg, USA.

Jun, S. P., Yoo, H. S., \& Choi, S. (2018). Ten years of research change using Google Trends: From the perspective of big data utilizations and applications. Technological Forecasting and Social Change, 130, 69-87. https://doi.org/10.1016/j.techfore.2017.11.009

Kahn, J. R., \& Pearlin, L. I. (2006). Financial strain over the life course and health among older adults. Journal of Health and Social Behavior, 47, 17-31. https://doi.org/10.1177/002214650604700102

Kalkhoran, S., Berkowitz, S. A., Rigotti, N. A., \& Baggett, T. P. (2018). Financial strain, quit attempts, and smoking abstinence among U.S. adult smokers. American Journal of Preventative Medicine, 55(1), 80-88. https://doi.org/10.1016/j.amepre.2018.01.036

Kaplan, R. M., \& Saccuzzo, D. P. (2017). Psychological Testing: Principles, Applications, and Issues (9th ed.) Boston, MA: Cengage Learning.

Kempson, E., Finney, A., \& Poppe, C. (2017). Financial well-being, a conceptual model and preliminary analysis. (SIFO Working Paper No. 3-2017). Oslo and Akershus University College of Applied Sciences https://doi.org/10.13140/RG.2.2.18737.68961

Kiely, K. M., Leach, L. S., Olesen, S. C., \& Butterworth, P. (2015). How financial hardship is associated with the onset of mental health problems over time. Social Psychiatry and Psychiatric Epidemiology, 50(6), 909-918. https://doi.org/10.1007/s00127-015-1027-0

Kim, J., \& Garman, E. T. (2003). Financial Stress and absenteeism: An empirically derived model. Journal of Financial Counseling and Planning, 14(1), 31-42.

Kimberlin, C. L., \& Winterstein, A. G. (2008). Validity and reliability of measurement instruments used in research. American Journal of Health-System Pharmacy, 65(23), 2276-2284. https://doi.org/10.2146/ajhp070364

Klein, J. T. (1990). Interdisciplinarity: History, Theory, and Practice. Detroit, MI: Wayne State University Press.

Klein, J. T. (1996). Crossing Boundaries: Knowledge, Discplinarities, and Interdisciplinarities. Charlottesville, VA: University Press of Virginia.

Klein, J. T. (2010). A taxonomy of interdisciplinarity. In R. Frodeman, J. T. Klein, \& C. Mitcham (Eds.) The Oxford Handbook of Interdisciplinarity. New York, NY: Oxford University Press Inc. pp.15-30.

Lajuni, N., Bujang, I., Karia, A. A., \& Yacob, Y. (2017). The role of educators and the influence of financial behavior on personal financial distress among undergrad students of public universities in Sabah, Malaysia. International Journal, 2(6), 121-130.

Latsou, D., Geitona, M. (2018). The effects of unemployment and economic distress on depression symptoms. Materia Socio Medica, 30(3), 180-184. https://doi.org/10.5455/msm.2018.30.180-184

Leal, R., Viseu, J. N., de Jesus, S. N., Paixão, O., \& Greenglass, E. (2014). Economic stressors and symptoms of psychological distress: data from a Portuguese sample. In K. Kaniasty, K. A. Moore, S. Howard, \& P. Buchwald (Eds.), Stress and Anxiety. Applications to health and well-being, work stressors and assessment (pp.17-23). Logos Verlag.

Lester, J. D., \& Lester, J. D. Jr. (2015). Writing Research Papers: A Complete Guide (15th ed.). USA: Pearson Education. 
Levit, T., \& Cismaru, M. (2020.) Marketing social marketing theory to practitioners. International Review on Public and Nonprofit Marketing, 17(2), 237-252. https://doi.org/10.1007/s12208-020-00245-4

Lim, H., Heckman, S., Montalto, C., \& Letkiewicz, J. (2014). Financial stress, self-efficacy, and financial help-seeking behavior of college students. Journal of Financial Counseling and Planning, 25(2), 148-160.

Madianos, M., Economou, M., Alexiou, T., \& Stefanis, C. (2011). Depression and economic hardship across Greece in 2008 and 2009: Two cross-sectional surveys nationwide. Social psychiatry and psychiatric epidemiology, 46(10), 943-952. https://doi.org/10.1007/s00127-010-0265-4

Mann, F. D., Krueger, R. F., \& Vohs, K. D. (2020). Personal economic anxiety in response to COVID-19. Personality and Individual Differences, 167, 110233. https://doi.org/10.1016/j.paid.2020.110233

Marjanovic, Z., Greenglass, E., Fiksenbaum, L., \& Bell, C. (2013). Psychometric evaluation of the Financial Threat Scale (FTS) in the context of the great recession. Journal of Economic Psychology, 36, 1-10. https://doi.org/10.1016/j.joep.2013.02.005

McCarthy, Y. (2011). Behavioural Characteristics and Financial Distress (European Central Bank Working Paper, No. 1303), Germany: European Central Bank.

Mills, R. J., Grasmick, H. G., Morgan, C. S., \& Wenk, D. (1992). The effects of gender, family satisfaction, and economic strain on psychological well-being. Family Relations, 41(4), 440-445. https://doi.org/10.2307/585588

Muckler, F. A., \& Seven, S. A. (1992). Selecting performance measures:" Objective" versus" subjective" measurement. Human Factors, 34(4), 441-455. https://doi.org/10.1177/001872089203400406

Muir, K., Reeve, R., Connolly, C., Marjolin, A., Salignac, F., \& Ho, K. (2016). Financial resilience in Australia 2015. Centre for Social Impact (CSI), University of New South Wales, for National Australia Bank.

Netemeyer, R. G., Warmath, D., Fernandes, D., \& Lynch, J. (2017). How Am I Doing? Perceived Financial Well-Being, Its Potential Antecedents, and Its Relation to Overall Well-Being. Journal of Consumer Research, 45(1), 68-89. https://doi.org/10.1093/jcr/ucx109

Newman, I., Ridenour, C., Weis, D. M., \& McNeil, K. (1997). Theses and dissertations: A guide to writing in social and physical sciences. Educational Leadership Faculty Publications, 82, 13-22.

O'Neill, B., Prawitz, A., Sorhaindo, B., Kim, J., \& Garman, E. T. (2006). Changes in health, negative financial events, and financial distress/financial well-being for debt management program clients. Journal of Financial Counseling and Planning, 17(2), 46-63. https://doi.org/10.1037/t60365-000

O'Neill, B., Sorhaindo, B., Xiao, J. J., \& Garman, E. T. (2005). Financially distressed consumers: Their financial practices, financial well-being, and health. Journal of Financial Counseling and Planning, 16(1), 73-87.

Park, N., Heo, W., Ruiz-Menjivar, J., \& Grable, J. E. (2017). Financial Hardship, Social Support, and Perceived Stress. Journal of Financial Counseling and Planning 28(2), 322-332. https://doi.org/10.1891/1052-3073.28.2.322

Pearlin, L. I., Menaghan, E. G., Lieberman, M. A., \& Mullan, J. T. (1981). The stress process. Journal of Health and Social Behavior, 22(4), 337-356. https://doi.org/10.2307/2136676

Perry, L. M., Hoerger, M., Seibert, K., Gerhart, J. I., O’Mahony, S., \& Duberstein, P. R. (2019). Financial strain and physical and emotional quality of life in breast cancer. Journal of Pain and Symptom Management, 58(3), 454-459. https://doi.org/10.1016/j.jpainsymman.2019.05.011

Perry-Rivers, P. (2014). Stratification, economic adversity, and entrepreneurial launch: The effect of resource position on entrepreneurial strategy. Entrepreneurship Theory and Practice, 40(3), 685-712. https://doi.org/10.1111/etap.12137

Prawitz, A. D., Garman, E. T., Sorhaindo, B., O’Neill, B., Kim, J., \& Drentea, P. (2006a). In Charge Financial Distress/Financial Well-Being scale: Development, administration, and score interpretation. Journal of Financial Counseling and Planning, 17(1), 34-50. https://doi.org/10.1037/t60365-000

Price, R. H., Choi, J. N., \& Vinokur, A. D. (2002). Links in the chain of adversity following job loss: How financial strain and loss of personal control lead to depression, impaired functioning, and poor health. Journal of Occupational Health Psychology, 7(4), 302-312. https://doi.org/10.1037//1076-8998.7.4.302

Seymour Management Consulting Inc. (2017). Focusing on the financial health and resilience of Canadians. Part 1- Focusing on financial wellness: A paradigm shift. Retrieved form 
http://financialhealthindex.org/of_files/focusing-financial-health-resilience-canadians-white-paper-part-1/

Shaffer, J. A., DeGeest, D., \& Li, A. (2016). Tackling the problem of construct proliferation: A guide to assessing the discriminant validity of conceptually related constructs. Organizational Research Methods, 19(1), 80-110. https://doi.org/10.1177/1094428115598239

Simon, H. A. (2014). Information-Processing explanations of understanding. In P. W. Jusczyc \& R. M. Klein (Eds.), The nature of thought: essays in honor of D.o. Hebb, 1980, Routledge, Taylor \& Francis, Psychology Press.

Sinclair, R. R., \& Cheung, J. H. (2016). Money Matters: Recommendations for financial stress research in occupational health psychology. Stress and Health, 32, 181-193. https://doi.org/10.1002/smi.2688

Starrin, B., Åslund, C., \& Nilsson, K. W. (2009). Financial stress, shaming experiences and psychosocial ill-health: Studies into the finances-shame model. Social Indicators Research, 91(2), 283-298. https://doi.org/10.1007/s11205-008-9286-8

Tormos, R. (2019). Measuring personal economic hardship and its impact on political trust during the great recession. Social Indicators Research, 144, 1209-1232. https://doi.org/10.1007/s11205-019-02082-3

Turcotte, M., \& Hango, D. (2020). Impact of economic consequences of COVID19 on Canadians' social concerns (Statistics Canada Working Paper No. 4528001). Retrieved from https://www150.statcan.gc.ca/n1/en/pub/45-28-0001/2020001/article/00025-eng.pdf?st=hvGbddj2

Vinokur, A., \& Caplan, R. D. (1987). Attitudes and social support: Determinants of job-seeking behavior and well-being among the unemployed. Journal of Applied Social Psychology, 17(12), 1007-1024. https://doi.org/10.1111/j.1559-1816.1987.tb02345.x

Viseu, J., Leal, R., Neves de Jesus, S., Pinto, P., Pechorro, P., \& Greenglass, E. (2018). Relationship between economic stress factors and stress, anxiety, and depression: Moderating role of social support. Psychiatry Research, 268, 102-207. https://doi.org/10.1016/j.psychres.2018.07.008

Voydanoff, P. (1990). Economic distress and family relations: A review of the eighties. Journal of Marriage and the Family, 52(4), 1099-1115. https://doi.org/10.2307/353321

Wharton, B. I. (2007). First-year student financial behavior and academic success (Unpublished doctoral dissertation). The Ohio State University, Columbus, USA.

Witteveen, D. (2020). Sociodemographic inequality in exposure to COVID-19-inde economic hardship in the United Kingdom. Research in Social Stratification and Mobility, 69, 100551. https://doi.org/10.1016/j.rssm.2020.100551

Zagorsky, J. L. (2007). Do you have to be smart to be rich? The impact of IQ on wealth, income and financial distress. Intelligence, 35(5), 489-501. https://doi.org/10.1016/j.intell.2007.02.003

Zheng, L., Xu, X., Xu, T., Yang, L., Gu, X., \& Wang, L. (2019). Financial strain and intimate partner violence against married women in postreform China: Evidence from Chengdu. Journal of Interpersonal Violence, 1-22. https://doi.org/10.1177/0886260519853406

\section{Copyrights}

Copyright for this article is retained by the author(s), with first publication rights granted to the journal.

This is an open-access article distributed under the terms and conditions of the Creative Commons Attribution license (http://creativecommons.org/licenses/by/4.0/). 